\title{
The Chinese medicine JC-001 enhances the chemosensitivity of Lewis lung tumors to cisplatin by modulating the immune response
}

\author{
Meng-Hsien Chuang ${ }^{1}$, Ming-Shiou Jan ${ }^{2,3,4}$, Jinghua Tsai Chang ${ }^{1,5^{*}+}$ and Fung-Jou Lu ${ }^{1,6^{*}+}$
}

\begin{abstract}
Background: JC-001 is a Chinese medicine that can modulate the immunity in Hepa 1-6 tumor-bearing mice, and we questioned whether JC-001 can serve as efficient adjuvant chemotherapy. We aimed to identify a novel approach for enhancing cis-diamminedichloroplatinum (II) (CDDP)-based chemotherapy by immunomodulation.

Methods: The anti-tumor activity in vitro was determined based on foci formation and a 3-(4,5-dimethylthiazol-2-yl)2,5-diphenyltetrazolium bromide (MTT) assay. A LLC1 tumor xenograft model was used to analyze the activity of tumor rejection in vivo. The tumors were analyzed through hematoxylin and eosin (H\&E) staining, immunohistochemistry (IHC) staining and cytokine arrays.

Results: JC-001 suppressed foci formation and reduced the viability of Lewis lung carcinoma (LLC1) cells in vitro. JC-001 suppressed LLC1 tumor growth in immunodeficient BALB/C nude mice and in immunocompetent C57BL/6 mice to an even greater extent. Furthermore, JC-001 up-regulated interferon- $\gamma$ expression in the tumor microenvironment, enhanced the Th1 response in tumor-bearing mice, and increased the chemosensitivity of LLC1 tumors to CDDP chemotherapy. The results of our study suggest that JC-001 is associated with low cytotoxicity and can significantly suppress tumor growth by enhancing the Th1 response.
\end{abstract}

Conclusion: JC-001 is a Chinese medicine with potential clinical applications in CDDP-based chemotherapeutic regimens.

Keywords: CDDP, Lung cancer, Immune, Chemosensitivity

\section{Background}

Lung cancer is the leading cause of cancer-related deaths worldwide [1]. Lung cancer is generally classified into 2 major types, with $15 \%$ of cases classified as small-cell lung cancer (SCLC) and 85\% of cases classified as non-smallcell lung cancer (NSCLS). NSCLC can be further divided into the adenocarcinoma (ADCA), squamous cell carcinoma (SCCA), large-cell carcinoma and bronchoalveolar cell carcinoma subtypes [2]. Contributing factors to the pathogenesis of lung cancer include tobacco smoke, genetic aberrations, hormonal factors, chronic obstructive

\footnotetext{
* Correspondence: jinghuat@csmu.edu.tw; fjlu@csmu.edu.tw

${ }^{\dagger}$ Equal contributors

'Institute of Medicine, Chung Shan Medical University, No. 110, Sec. 1,

Jianguo N. Rd, Taichung City 40201, Taiwan

Full list of author information is available at the end of the article
}

pulmonary disease, and viral infection (human papillomavirus), which ultimately induce tissue damage and changes to the microenvironment [3-6]. Current first-line therapies usually comprise a combination of chemotherapies and targeted therapies directed towards 1 or more of the various signaling pathways implicated in cancer. However, the 5 -year survival rate of lung cancer is less than 20\% [1]; therefore, more effective therapies are needed.

cis-diamminedichloroplatinum (II) (cisplatin; CDDP) is an inorganic compound that has been used to treat malignant tumors over the last 4 decades. However, the side effects of CDDP, including vomiting, digestive tract disorders, ototoxicity and nephrotoxicity, limit its clinical applications [7]. Notably, nephrotoxicity is the primary dose-limiting side effect, as CDDP accumulates in the kidney where it can cause excessive injury to the proximal 
renal tubule cells [8]. To improve the therapeutic efficacy and minimize toxicity, CDDP is often combined with other chemotherapies or targeted agents. However, the clinical outcomes and quality of life of patients receiving CDDP remains far from ideal [9-11].

In a previous study, we demonstrated that JC-001 suppresses Hepa 1-6 murine tumor cell growth in C57BL/6 mice by modulating the immune response and upregulates Hepa 1-6-specific cytotoxicity in Hepa 1-6 cells co-cultured with Hepa 1-6-immunized splenocytes [12]. In this study, we evaluated whether JC-001 can suppress the growth of Lewis lung carcinoma cell line (LLC1)-derived tumors and increase the efficacy of CDDP by enhancing its immunomodulatory effects.

\section{Methods}

Antibodies, chemicals and reagents

Anti-mouse CD8 (BS-0648R) and anti-IFN- $\gamma$ (BS-0480R) antibodies were purchased from Bioss (Boston, MA, United States). The anti-IL-12 p70 antibody (NBP185564) was purchased from Novus Biological (Littleton, MA, United States). Dulbecco's Modified Eagle Medium (DMEM) culture medium, Roswell Park Memorial Institute (RPMI) medium, L-glutamine, trypsin-EDTA and fetal bovine serum were purchased from Gibco (Grand Island, NY, United States). ELISPOT assay kits for mouse IL-2, IL-10, IL-17A, TNF- $\alpha$, TGF- $\beta$ and IFN- $\gamma$ were purchased from eBioscience (San Diego, CA, United States). Cisplatin was purchased from Sigma-Aldrich (Stockholm, Sweden). Chloral hydrate was purchased from SigmaAldrich (Stockholm, Sweden). QuantiChrom ${ }^{\text {ma }}$ Creatinine Assay Kit, QuantiChrom ${ }^{\text {TM }}$ Urea Assay Kit, EnzyChrom ${ }^{\mathrm{Ts}}$ Alanine Transaminase Assay Kit, EnzyChrom ${ }^{\mathrm{Tm}}$ Aspartate Transaminase Assay Kit and QuantiChrom ${ }^{\mathrm{Tm}}$ BCG Albumin Assay Kit were purchased from BioAssay Systems (Hayward, CA, United States).

\section{Extraction of plant materials}

The Chinese herbal medicine JC-001 was provided by Jun Chen Biotech Co., Ltd., Taiwan. The primary components of this concoction are Bupleurum chinense DC (8\%), Gentiana scabra Bge. (16\%), Rheum palmatum L. (8\%), Clematis montana Buch.-Ham. (12\%), Carthamus tinctorius L. (8\%), Prunus persica (L.) Batsch (8\%), Angelica dahurica (Fisch. ex Hoffm.) Benth. et Hook. f. (8\%), Siegesbeckia orientalis L. (8\%), Glycyrrhiza uralensis Fisch. (8\%). and Solanum incanum L. (16\%). The JC-001 hot water extraction process followed our previously described protocol [12].

\section{Animals and xenograft tumor model}

In this study, we followed the guidelines of the Institutional Animal Care and Use Committee (IACUC) of Chung Shan Medical University (CSMU) for the care and use of experimental animals. Eight-week-old female C57BL/6 and male BALB/c nude mice weighing 20-25 g were obtained from BioLASCO (Taichung, Taiwan). The mice were maintained in a 12-h light/dark cycle. After 1 week of adaptation, the experimental procedures were initiated.

In total, $10^{5}$ LLC1 (ATCC number: CRL-1642; obtained from the Bioresource Collection and Research Center, Taiwan) cells were injected subcutaneously in each eightweek-old female C57BL/6 mouse. After three days, the mice were divided into control $(n=15), 1 \mathrm{X}$ JC-001 $(740 \mathrm{mg} / \mathrm{kg} ; n=14)$ and 3X JC-001 $(2467 \mathrm{mg} / \mathrm{kg} ; n=13)$ groups and fed with $200 \mu \mathrm{L}$ of JC-001 or autoclaved double-distilled water daily. At day 23 after tumor inoculation, the mice were anesthetized with a single intraperitoneal injection of $400 \mathrm{mg} / \mathrm{kg}$ chloral hydrate, and the tumors were surgically excised, imaged, weighed and fixed in $10 \%$ formalin.

\section{$B A L B / c$ nude (immunodeficient) mouse xenograft model}

Seven-week-old immunodeficient BALB/c nude mice were housed in individual ventilated cages for 1 week prior to the injection of cancer cells. To prepare the cells for inoculation, $100 \mu \mathrm{L}$ of RPMI containing $5 \times 10^{5}$ LLC1 cells was combined with $100 \mu \mathrm{L}$ of Matrigel $(10 \mathrm{mg} / \mathrm{mL})$. Each mouse was subcutaneously inoculated with $200 \mu \mathrm{L}$ of cells/Matrigel solution. On the second day after inoculation, the mice were divided into control $(n=8)$ and 3X JC-001 groups $(n=8)$, and fed with $200 \mu \mathrm{L}$ of $2467 \mathrm{mg} / \mathrm{kg}$ JC-001 or autoclaved doubledistilled water. On day 26 after injection, the mice were anesthetized, and the tumors were surgically excised, imaged, weighed and fixed in $10 \%$ formalin.

\section{Foci-formation assay}

For the foci-formation assay, 200 LLC1 cancer cells were seeded in a $3.5-\mathrm{cm}$ plate and incubated for $24 \mathrm{~h}$ in a $37^{\circ} \mathrm{C}$ incubator with $5 \% \mathrm{CO}_{2}$. The medium was discarded and replaced with $3 \mathrm{~mL}$ of fresh medium containing 0 (blank), $50,100,200,400$ or $800 \mu \mathrm{g} / \mathrm{mL}$ JC-001. The cells were incubated for approximately 7-9 days without disturbance. When the blank (control) cells formed visible colonies, the cells were washed with $1 \times$ PBS and fixed with $100 \%$ ethanol for $20 \mathrm{~min}$. After removing the ethanol, the cells were stained with $20 \%$ Giemsa for $30 \mathrm{~min}$ at room temperature. After the cells were rinsed with water, colony morphology was evaluated, and the number of colonies was counted. These experiments were conducted in triplicate.

\section{Cell viability assay}

The cells $\left(2 \times 10^{4}\right)$ were seeded into 24-well dishes with $1 \mathrm{~mL}$ of culture medium and incubated overnight to permit cell adhesion. Then, the medium was refreshed with medium containing $0-800 \mu \mathrm{g} / \mathrm{mL} \mathrm{JC-001}(n=4$ samples at each concentration). After $96 \mathrm{~h}$ of treatment, $400 \mu \mathrm{L}$ 
of 3-(4,5-dimethylthiazol-2-yl)-2,5-diphenyltetrazolium bromide (MTT) reagent was added to each well at a final concentration of $500 \mu \mathrm{g} / \mathrm{mL}$. After four hours of reaction, the reagent was removed, and DMSO was added to dissolve the product. Cell viability was determined by comparing the $\mathrm{OD}_{470}$ with that of the control group. These experiments were conducted in triplicate.

\section{Tumor microenvironment analysis}

The RayBio ${ }^{\circ}$ Mouse Cytokine Antibody Array III kit (RayBiotech, Norcross, GA, United States) was used to detect cytokine changes in tumor tissues. The tumor tissues were frozen in liquid nitrogen and then homogenized in $10 \mathrm{~mL}$ of lysis buffer at room temperature for $10 \mathrm{~min}$. The homogenized solution was then centrifuged at $16000 \mathrm{rpm}$ at $4{ }^{\circ} \mathrm{C}$ for $2 \mathrm{~h}$. The supernatant was transferred to a new tube, and the concentration was adjusted to $1 \mathrm{mg} / \mathrm{mL}$. A volume of $1 \mathrm{~mL}$ of the lysate was added to the RayBio ${ }^{\circ}$ Mouse Cytokine Antibody Array III, and the chip was incubated at $4{ }^{\circ} \mathrm{C}$ overnight with gentle shaking. The chip was then washed with wash buffer I 3 times for $10 \mathrm{~min}$ each and subsequently washed with wash buffer II 3 times for 10 min each. The chip was incubated with biotinylated primary antibodies. After washing, the chip was incubated with streptavidinconjugated secondary antibody. The serum was diluted 1:4 in blocking buffer and directly added to the RayBio ${ }^{\circ}$ Mouse Cytokine Antibody Array III to allow for binding.

\section{Co-culture condition and cytokine analysis}

To generate the LLC1-immunized splenocytes, C57BL/6 mice were immunized by injection (s.c.) with $5 \times 10^{3}$ LLC1 cells at days 0, 7 and 14. After immunization, the mice were sacrificed, and the spleens were removed aseptically using a routine surgical procedure at day 21. Single-cell suspensions were prepared by filtering the spleen tissue through mesh screens. The erythrocytes were lysed, and the cells were cultured in RPMI-1640 supplemented with 10\% FBS and $1 \%$ penicillin/streptomycin.

LLC1-immunized splenocytes $\left(4 \times 10^{6} /\right.$ well $)$ were cocultured with LLC1 cells $\left(1 \times 10^{5} /\right.$ well $)$ in 24 -well plates, and $2 \mathrm{ml}$ of RPMI-1640 supplemented with 10\% FBS and $1 \%$ penicillin/streptomycin was added to each well. After a 48-h incubation, the culture medium was collected, and cytokine secretion was analyzed using the ELISPOT assay. All reagents used in the ELISPOT assay were supplied in the assay kit, and the assay was conducted according to the manufacturer's instructions. These experiments were conducted in triplicate.

CDDP and JC-001 combination therapy in immunocompetent LLC1 tumor model

Eight-week-old C57BL/ 6 female mice were randomly divided into 5 groups: the naive $(n=6)$, control $(n=16)$,
CDDP $(n=16)$, JC-001 $(n=16)$ and JC-001/CDDP $(n=16)$ groups. All mice were subcutaneously injected with $1 \times 10^{5}$ LLC1 cells. Two days after the inoculation, the mice were fed $200 \mu \mathrm{L}$ of $\mathrm{H}_{2} \mathrm{O}$ or $2467 \mathrm{mg} / \mathrm{kg} \mathrm{JC}-001$ for 18 days. On day 3, 6, 9, 12, 15 and 18 after the inoculation, the mice were treated with $1 \mathrm{mg} / \mathrm{kg}$ CDDP (i.p.) or normal saline. On day 22, the mice were anesthetized, and the tumors were surgically excised, imaged, weighed and fixed in 10\% formalin. Serum levels of creatinine, urea, alanine aminotransferase (ALT), aspartate transaminase (AST) and albumin were determined by AllBio Science Inc. (Taichung, Taiwan).

\section{Histological and immunohistochemistry analysis}

All the histological work by Rapid Science Co, Ltd., Taichung, Taiwan. And the qualitative analyzed by Prof. Chung-Hung Tsai, Department of Pathology, Chung Shan Medical University Hospital. Both Rapid Science Co, Ltd. and Prof. Chung-Hung Tsai were blind for all the sample. Tumor tissues extracted from the tumor-bearing mice were fixed in $4 \%$ formaldehyde buffered with phosphate solution (0.1 mol/L; pH 7.4) and processed for paraffin embedding. The paraffin blocks were sliced into $3-\mu \mathrm{m}$ sections, stained with hematoxylin and eosin (H\&E) and examined using light microscopy. For the immunohistochemistry (IHC) analysis, the slides were deparaffinized in xylene and rehydrated in decreasing concentrations of alcohol. Antigen retrieval was conducted by heating the tissues in a microwave. Then, 3\% hydrogen peroxide was used to inactivate endogenous peroxidase, and the samples were blocked with $5 \%$ bovine serum in PBS. The slides were incubated at $4{ }^{\circ} \mathrm{C}$ overnight with the anti-mouse CD8 (1:400), anti-IL-12 p70 (1:100) or anti-IFN- $\gamma(1: 200)$ primary antibodies. After the slides were washed with PBST, they were sequentially incubated with biotinylated goat anti-rabbit IgG secondary antibody, streptavidin-biotin complex, and $3,3^{\prime}$-diaminobenzidine for $20 \mathrm{~min}$ at room temperature.

\section{Statistical analyses}

Statistical analyses of the in vitro experimental data were conducted using the two-tailed Student's t-test for simple comparisons between 2 values, and values are presented as the mean \pm standard deviation. For the in vivo experimental data, the unpaired Student's t-test was used to compare the means between 2 groups. A $p$ value $<0.05$ was considered statistically significant. All the data were used the GraphPad Prism 5.0 to calculate and statistical analyses.

\section{Results}

\section{JC-001 inhibited foci formation and disrupted cell viability of LLC1 cells}

To characterize the role of JC-001 in foci formation, LLC1 cells were treated with 0 (blank), 50, 100, 200, 400 or 
$800 \mu \mathrm{g} / \mathrm{mL}$ JC-001. The number of foci decreased with increasing concentrations of JC-001, with an $\mathrm{IC}_{50}$ of $325 \mu \mathrm{g} / \mathrm{mL}$ (Fig. 1a). In addition to the decrease in colony size and number, JC-001 treatment also rendered the colonies less adherent, indicating that JC-001 might affect cell-cell interactions. To determine whether the inhibition of foci formation was due to a disruption of cell viability, LLC1 cells were treated with 0 (blank), 50, 100, 200, 400 or $800 \mu \mathrm{g} / \mathrm{mL} \mathrm{JC}-001$, and the MTT assay was conducted $96 \mathrm{~h}$ after the treatment. We found that $0-400 \mu \mathrm{g} / \mathrm{mL} \mathrm{JC}$ -
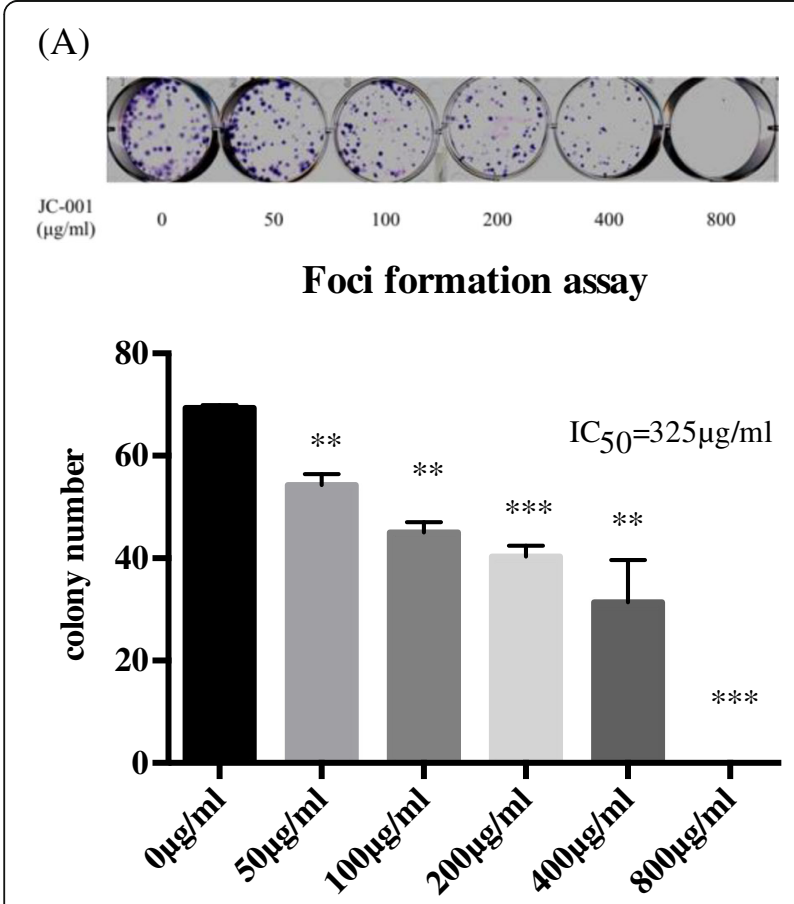

(B)

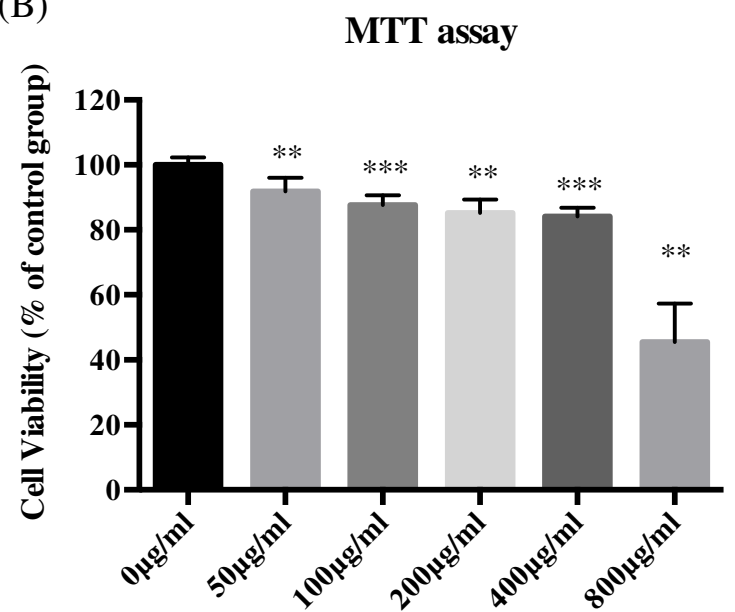

Fig. 1 JC-001 affects the foci formation activity and cell viability of LLC1 cells in vitro. a The IC $C_{50}$ of JC-001 for the inhibition of foci formation by LLC1 was $325 \mu \mathrm{g} / \mathrm{mL}$. $\mathbf{b}$ The effect of JC-001 on the cell viability of LLC1. ${ }^{*} p<0.05,{ }^{* *} p<0.01,{ }^{* * *} p<0.001$ compared with the control group. The results are expressed as mean \pm SD of three separate experiments
001 inhibited cell viability by approximately $10 \%$, whereas JC-001 at a concentration of $800 \mu \mathrm{g} / \mathrm{mL}$ inhibited cell viability by $55 \%$ (Fig. 1b). The observation that $100 \mu \mathrm{g} / \mathrm{mL}$ JC-001 inhibited foci formation but not cell viability suggested that JC-001 inhibits foci formation in LLC1 cells in a growth-independent manner.

The anti-tumor activity of JC-001 in the immunocompetent xenograft tumor model

As JC-001 has the ability to inhibit both foci formation and growth of LLC1 cells, we investigated whether JC-001 exerts anti-tumor activity in an LLC1 xenograft model. The immunocompetent C57BL/6 mice were subcutaneously injected with $1 \times 10^{5}$ LLC1 cells. Three days after the inoculation, the mice were treated with $200 \mu \mathrm{L}$ of $\mathrm{H}_{2} \mathrm{O}, 1$ X JC-001 $(740 \mathrm{mg} / \mathrm{kg})$ or 3X JC-001 (2467 mg/kg) daily. After 20 days of treatment, the tumor mass in mice treated with 1 X JC-001 or 3X JC-001 decreased by $59 \%$ and $86 \%$, respectively, compared with the $\mathrm{H}_{2} \mathrm{O}$ control group (Fig. 2). In addition, hepatosplenomegaly and renal atrophy were observed in mice inoculated with LLC1 cells, and the severity of these defects was reduced in the JC001-treated groups compared with the control group. These results indicated that JC-001 effectively inhibited tumor progression in immunocompetent mice.

The effect of JC-001 on cytokine expression in conditioned medium from a LLC1/splenocyte co-culture To characterize the function of JC-001 in the tumor microenvironment, we immunized C57BL/6 mice with $5 \times 10^{3}$ LLC1 cells to generate LLC1-immunized splenocytes. We co-cultured the effector (LLC1 immunized splenoctyes) and target cells (LLC1 cells) and analyzed cytokine secretion in the culture medium after $48 \mathrm{~h}$ of JC001 treatment. We found that JC-001 up-regulated the expression of IL-2, IL-10, TNF- $\alpha$, and IFN- $\gamma$ and downregulated the expression of TGF- $\beta$ and IL-17A in the conditioned medium (Fig. 3). The expression patterns of TNF- $\alpha$, IFN- $\gamma$ and IL-17 were consistent with the results of the cytokine array assay (Additional file 1) used to evaluate the tumor microenvironment. These findings indicated that JC-001 enhanced theTh1 response and inhibited the Th17 response.

\section{JC-001 enhanced chemosensitivity to CDDP in an} immunocompetent LCC1 xenograft tumor model

The significant nephrotoxicity associated with CDDP limits its clinical applications as an anti-tumor agent. Therefore, we evaluated whether JC-001 treatment could enhance the anti-tumor activity of low-dose CDDP chemotherapy.

There were no differences in the average tumor weight in mice injected with $1 \mathrm{mg} / \mathrm{kg}$ CDDP six times and the control group. A total of $12.5 \%$ of mice in this group did 

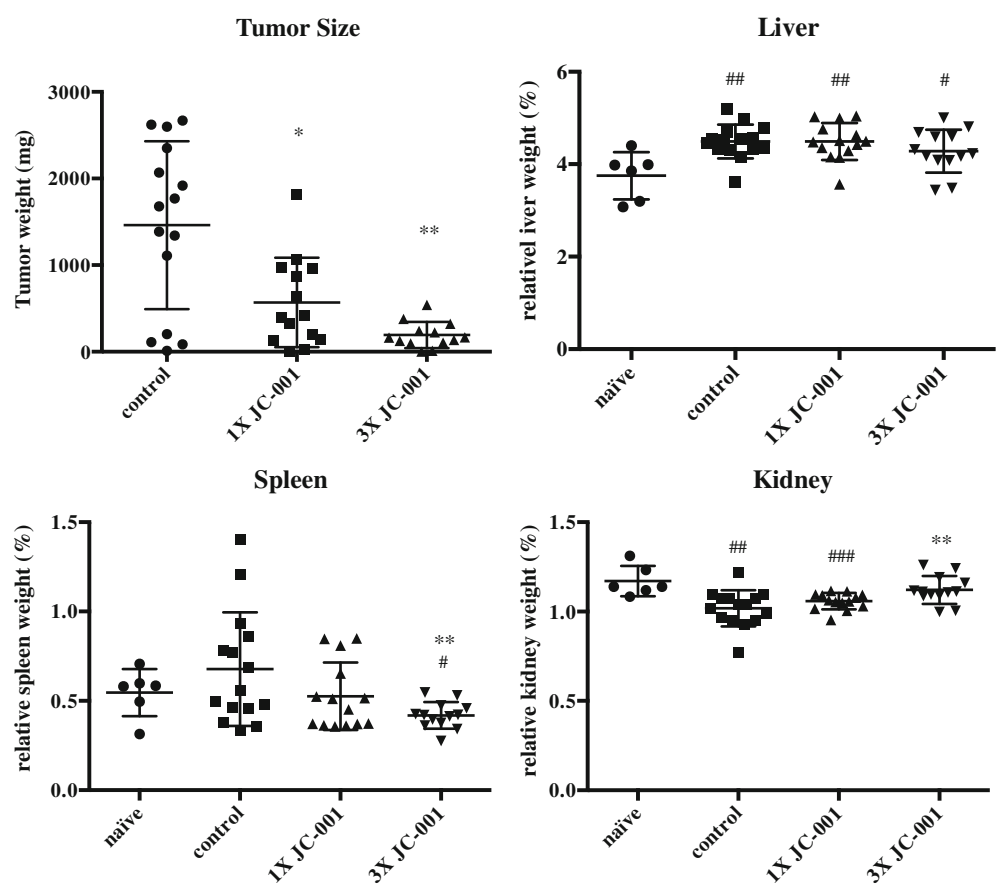

Fig. 2 JC-001 inhibits tumor growth and prevents organ damage in LLC1-inoculated C57BL/6 immunocompetent mice. After LLC1 tumor growth for 23 days, the average tumor weight in the control group reached $1461 \mathrm{mg}$, and the relative organ weights differed from those in the naiive group. The average tumor weight in the $3 \mathrm{XJC}$-001-treated group was significantly reduced to $193 \mathrm{mg} .{ }^{*} p<0.05,{ }^{* *} p<0.01,{ }^{* * *} p<0.001$ compared with the control group. \#p < 0.05, \#\#p <0.01, \#\#p < 0.001 compared with the naive group

not develop tumors (Fig. 4), which was a slight increase from the $6.25 \%$ observed in the control group. The average tumor weight decreased by $41 \%$ in the JC-001treated group compared with the control group, but the percentage of non-tumor-bearing mice was similar to the control group. In mice treated with CDDP in combination with 3X JC-001, a 33\% reduction in the average tumor weight was observed, and the percent of nontumor bearing mice increased to $50 \%$. Serum creatinine, urea, AST and albumin did not differ significantly among the groups. In two mice in the CDDP group and JC-001/CDDP group, serum creatinine reached $6.58 \mathrm{mg} /$ $\mathrm{dL}$ and $3.30 \mathrm{mg} / \mathrm{dL}$, respectively (Table 1 ). In addition, serum ALT in the control group and CDDP group exhibited a significant upward trend compared with the naive group, but this trend was not observed in the JC001 group and JC-001/CDDP group. Furthermore, an increase in immune cell infiltration was observed in tumor tissues derived from the JC-001- and JC-001/CDDPtreated mice compared with the control group, as demonstrated by H\&E staining (Fig. 5). CD8-positive lymphocytes were also observed in tumor tissues derived from JC-001- and JC-001/CDDP-treated mice. IHC staining revealed that the expression of the Th1 cytokine IL-12 p70 and IFN- $\gamma$ were up-regulated in the JC001- and JC-001/CDDP-treated tumors. These findings indicated that JC-001 significantly improved the chemosensitivity of LCC1-derived tumors to CDDP by modulating the immune response and successfully reduced tumor formation by $50 \%$.

\section{Discussion}

The murine Lewis lung cancer cell line LLC1 was initially isolated from a spontaneous carcinoma in a C57BL/6 mouse. LLC1 is a fast-growing cell line with a high invasive capability in vitro. We used LLC1 cells to establish xenograft models in both immunocompetent and immunodeficient animals.

In the immunocompetent C57BL/6 xenograft model, JC-001 effectively inhibited LLC1 tumor growth. Treatment with 3X JC-001 $(2467 \mathrm{mg} / \mathrm{ml})$ reduced tumor weight by $86 \%$ compared with the $\mathrm{H}_{2} \mathrm{O}$ control treatment and prevented tumor formation entirely in 1 mouse. Although only a $50 \%$ reduction in tumor mass was observed in the group treated with 10X JC-001 $(7400 \mathrm{mg} / \mathrm{kg}), 3$ mice were tumor-free (data not show). As JC-001 inhibited foci formation in LLC1 cells, we sought to determine whether the anti-tumor activity of JC-001 was mediated by the inhibition of LLC1 cell growth or by an enhancement in immunity in C57BL/6 mice.

To further investigate the role of adaptive immunity in JC-001-mediated anti-tumor activity, we established an LLC1 xenograft model in immunodeficient mice (BALB/c nude mice). In mice treated with $3 \mathrm{X}$ JC-001, we observed 
IL-2

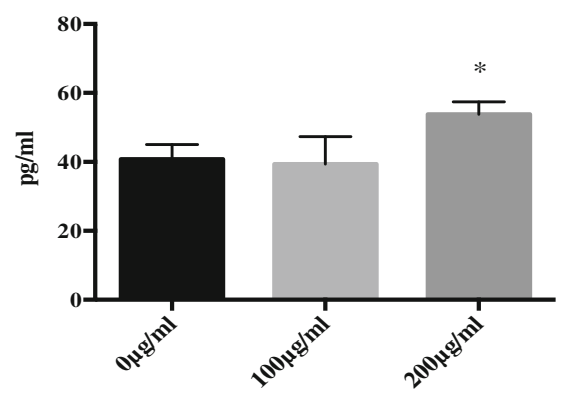

TNF- $\alpha$

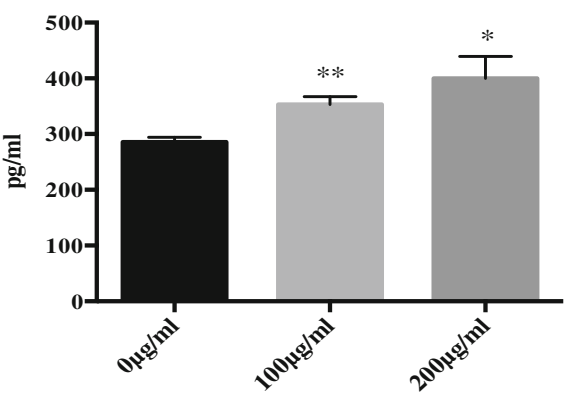

TGF- $\beta$

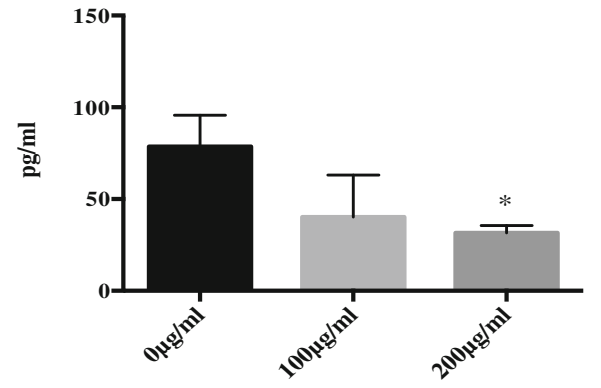

IL-10

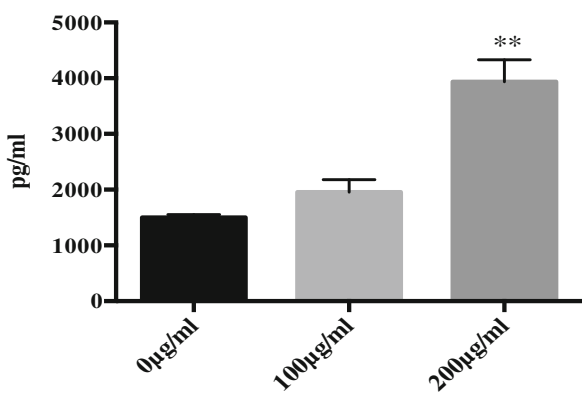

IFN- $\gamma$

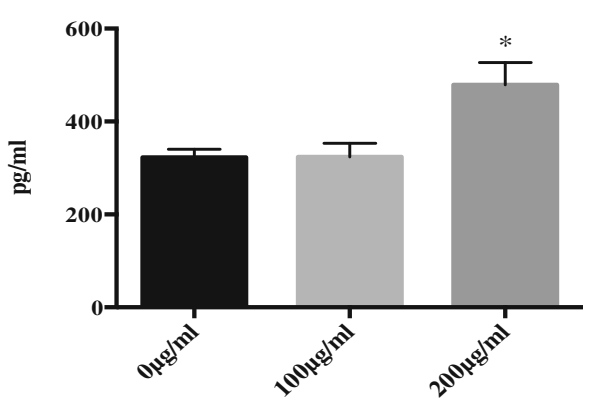

IL-17A

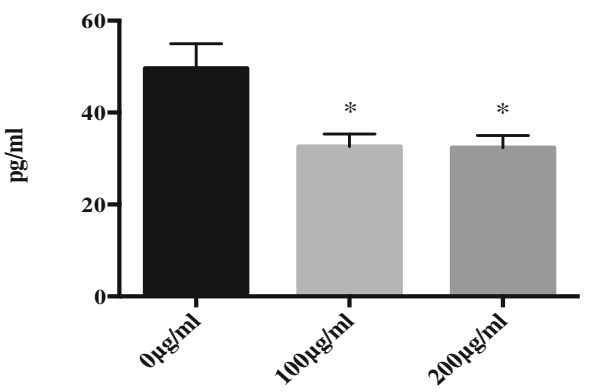

Fig. 3 JC-001 mediates cytokine secretion under co-culture conditions. The effector cell (LLC1 immunized splenocytes) to target cell (LLC1 cells) ratio was 40 , and each bar represents the mean \pm standard deviation $(n=3) .{ }^{*} p<0.05,{ }^{* *} p<0.01$ compared with the control group. After co-culture for 48 h, JC-001 up-regulated IL-2, IL-10, TNF- $\alpha$ and IFN- $\gamma$ and down-regulated IL-17A, and TGF- $\beta$ in the condition medium. The results are expressed as mean \pm SD of three separate experiments

a $61 \%$ reduction in tumor mass compared with the $\mathrm{H}_{2} \mathrm{O}$ control group (Additional file 1). Although BALB/c mice are T-cell deficient, they retain functional innate immunity. The $86 \%$ reduction in average tumor mass observed in C57BL/6 immunocompetent mice compared with the $61 \%$ reduction of tumor mass in BALB/c nude mice suggests that approximately $30 \%$ of the anti-tumor activity induced by $3 \mathrm{X}$ JC-001 in C57BL/6 mice resulted from the enhancement of the adaptive immune response. The remainder of the effect might have resulted from the enhancement of the innate immune response and/or the inhibition of LLC1 growth. Therefore, we cannot determine whether the anti-tumor activity of JC-001 in BALB/C nude mice was due to inhibiting LLC1 cell growth or due to an enhancement of the innate immune response.
Cytokines in the tumor microenvironment and in serum were further analyzed using the RayBio ${ }^{\circ}$ Mouse Cytokine Antibody Array III kit (Additional file 2). The expression of inflammatory-associated cytokines, including IL- $1 \alpha$, IL$1 \beta$ and IL-6, were significantly reduced in the tumor microenvironment of JC-001-treated mice, suggesting that JC-001 might decrease tumor-induced inflammation. JC001 treatment increased the expression of FasL and CX3CL1 (fractalkine) in the tumor microenvironment and in serum, whereas INF- $\gamma$ expression was elevated exclusively in the tumor microenvironment.

CX3CL1 can be expressed by cancer cells and endothelial cells. CX3CL1 is expressed as both the membrane-bound and secreted isoforms. CX3CR1, the receptor of CX3CL1, is expressed on the membrane of 

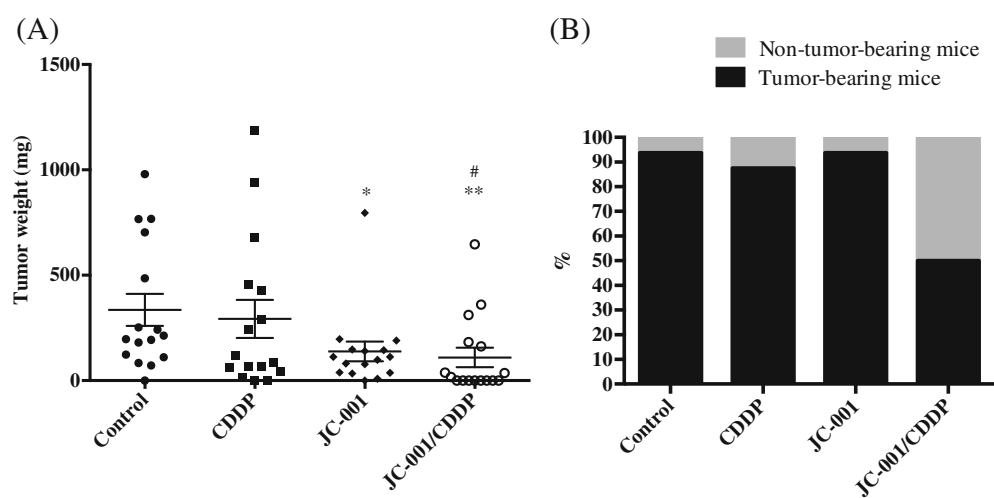

Fig. $4 \mathrm{JC}-001$ enhances the chemosensitivity of the LLC1 tumor model to CDDP. a Tumor mass. b Percentages of tumor/non-tumor-bearing mice in the groups $(n=16) .{ }^{*} p<0.05,{ }^{* *} p<0.01$ compared with the control group, \#p $<0.05$ compared with the CDDP-treated group. After JC-001/CDDP combined therapy, the percentage of non-tumor-bearing mice was significantly enhanced from $6.25 \%$ to $50 \%$ compared with the control group, $p=0.0031$

NK cells, CD14 monocytes, cytotoxic T cells and B cells. Thus, secreted CX3CL1 might act as a chemokine that induces infiltrating immune cells to attack tumor cells. Indeed, high levels of CX3CL1 are associated with a more favorable prognosis in patients with gastric adenocarcinoma and breast carcinoma [13, 14]. IFN- $\gamma$ enhances NK cell activity, antigen presentation, Th1 cell differentiation, and the activity of macrophages and cytotoxic T cells [15-18]. IFN- $\gamma$ not only induces cell cycle arrest [19-22] but also enhances class I major histocompatibility complex (MHC-I) expression on the surface of tumor cells, a phenomenon that can promote immune surveillance by triggering $\mathrm{CD}^{+} \mathrm{T}$ cell recognition [23]. Moreover, IFN- $\gamma$ might stimulate cells to express apoptosis-associated genes such as FasL. The results of the cytokine array revealed a cytokine signaling loop in the LLC1 xenograft model. The upregulation of CX3CL1 in the tumor microenvironment might attract infiltrating IFN- $\gamma$-expressing NK cells [18] that, in turn, stimulate the expression of FasL to activate apoptosis in tumor cells. Therefore, JC-001 appears to inhibit tumor progression by modulating the CX3CL1/ IFN- $\gamma /$ FasL signaling pathway.

In addition to activating anti-tumor cytokines, JC-001 inhibited the expression of pro-tumor cytokines, including leptin, LIX (CXCL5) and SCF, in both the tumor microenvironment and in serum. Leptin is an adipokine that plays an important role in energy homeostasis and regulates innate and adaptive responses, thereby modulating the tumor microenvironment [24, 25]. The leptin receptor (LEPR), which is expressed in multiple cancers, stimulates the growth and invasion of activated cancer cells and promotes angiogenesis [26, 27]. In addition, a recent study indicated that leptin promotes cancer stem cell survival [28]. The pro-angiogenic cytokine CXCL5 can also enhance the growth and invasion of cancer cells [29, 30]. High levels of CXCL5 correlate with a poor prognosis in patients with pancreatic cancer, colorectal cancer and hepatocellular carcinoma [31-33]. Thus, serum CXCL5 levels might represent a valuable prognostic factor. SCF and its receptor c-kit play an important role in the growth and survival of lung cancer stem cells [34]. Activation of the SCF/c-kit pathway enhances the growth and invasion of pancreatic cancer and colon cancer [35-37]. SCF induces mast cell infiltration and activation in tumors via mast cell-localized c-kit. The infiltrated mast cells secrete multiple pro-inflammatory factors and inhibit the activation of T cells and NK cells, thereby promoting tumor progression [38, 39]. Previous reports have demonstrated that an anti-SCF antibody enhances the sensitivity of cancer cells to chemotherapeutic agents $[40,41]$.

Table 1 Effect of JC-001/CDDP and combined treatment on serum creatinine, urea, ALT, AST and albumin

\begin{tabular}{llllll}
\hline & Naïve & Control & CDDP & JC-001 & JC-001/CDDP \\
\hline Creatinine $(\mathrm{mg} / \mathrm{dL})$ & $1.15 \pm 0.39$ & $1.27 \pm 0.41$ & $1.67 \pm 1.48$ & $1.14 \pm 0.49$ & $1.44 \pm 0.67$ \\
Urea $(\mathrm{mg} / \mathrm{dL})$ & $54.12 \pm 9.34$ & $54.79 \pm 6.25$ & $52.16 \pm 4.05$ & $47.55 \pm 9.29$ & $50.79 \pm 6.92$ \\
ALT $(\mathrm{U} / \mathrm{L})$ & $37.50 \pm 10.19$ & $75.79 \pm 50.91^{*}$ & $89.58 \pm 62.74^{*}$ & $52.66 \pm 29.25$ & $79.06 \pm 64.80$ \\
AST $(\mathrm{U} / \mathrm{L})$ & $245.6 \pm 23.2$ & $239.8 \pm 23.2$ & $238.4 \pm 27.9$ & $251.3 \pm 21.27$ & $250.5 \pm 34.4$ \\
Albumin $(\mathrm{g} / \mathrm{dL})$ & $0.49 \pm 0.07$ & $0.46 \pm 0.05$ & $0.47 \pm 0.04$ & $0.46 \pm 0.05$ & $0.47 \pm 0.04$ \\
\hline
\end{tabular}

${ }^{*} p<0.05$ compared with the naïve group 


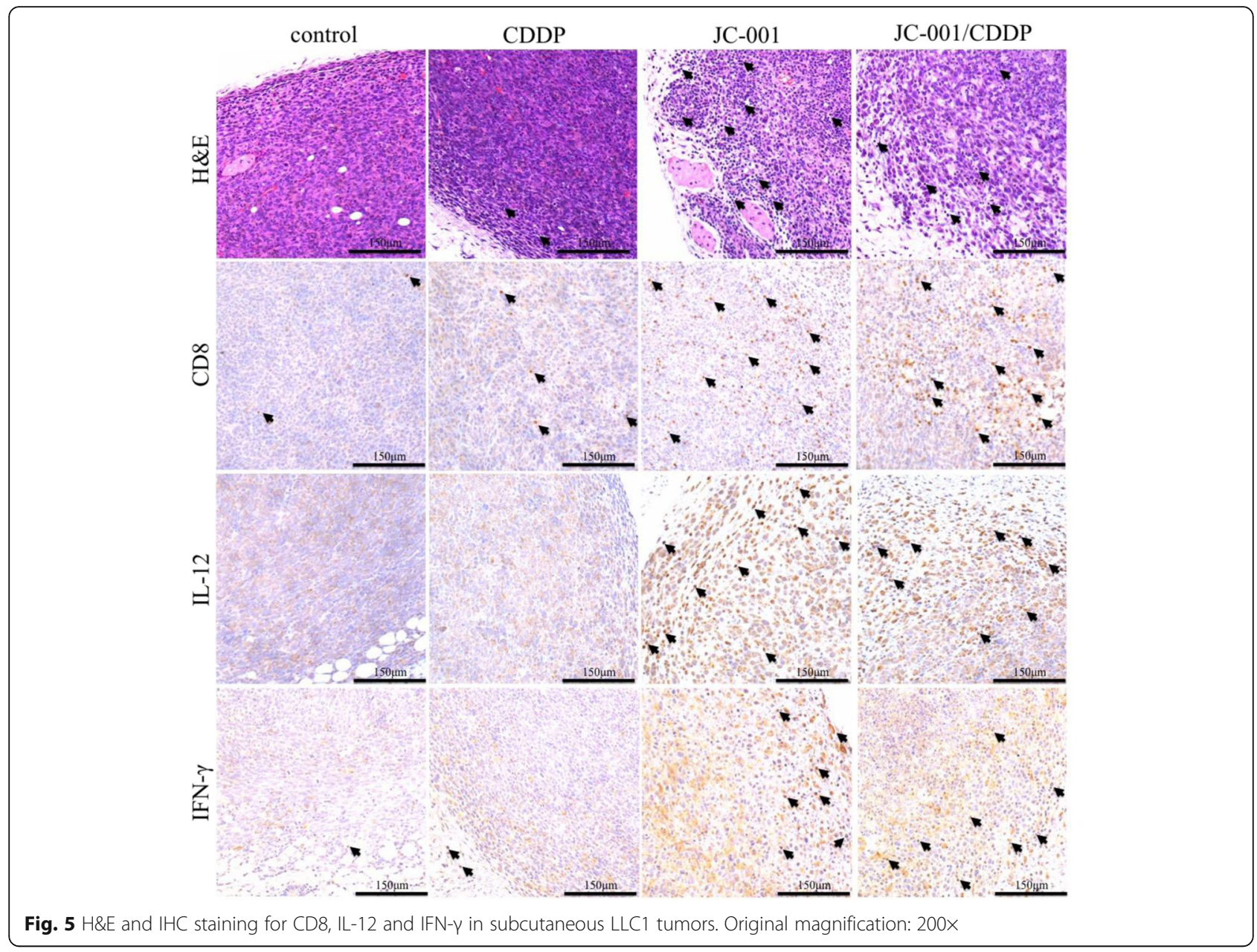

The variations in cytokine levels induced by JC-001 treatment suggested that JC-001 exerts its anti-tumor activity by enhancing the expression of anti-tumor cytokines and by reducing the expression of pro-tumor cytokines. Further investigation into the modulation of cytokine levels by JC-001 are needed to gain further insight into the molecular mechanisms underlying JC001-mediated anti-tumor activity. In addition, such studies might facilitate the development of molecular markers that exhibit similar variations in both the tumor microenvironment and serum and that can help predict a patient's response to JC-001.

IL-17A is produced by Th17 cells and promotes a protumor environment $[42,43]$. In the tumor microenvironment, IL-17A promotes neutrophil recruitment and DNA damage in local tissue by secreting radical oxygen species $[44,45]$. We previously reported that JC-001 down-regulates systemic Th17-mediated immunity in both Hepa 1-6 and LLC1 immunocompetent tumor models and up-regulates IL-10 secretion and downregulates IL-17A in conditioned medium from cocultured tumor cells and splenocytes. A growing body of evidence indicates that enhancing the immune response can increase the efficacy of chemotherapy [46-48]. The observation that JC-001 modulated the immune response indicates that JC-001 might enhance the clinical applications of CDDP. As the clinical application of CDDP is restricted by dose-limiting toxicity, we evaluated the effect of supplementing low dose CDDP treatment with JC-001. We found that JC-001 inhibited tumor formation by $50 \%$ and enhanced the Th1 response in the tumor microenvironment. These outcomes indicate that JC-001 can significantly enhance the anti-tumor effect of CDDP and avoid the toxicity caused by high doses, thereby expanding its potential clinical applications.

\section{Conclusion}

Our data revealed that JC-001 could increase CD8positive lymphocytes and enhance Th1 response in tumor microenvironment. Moreover, JC-001 significantly improved CDDP chemosensitivity and decreased CDDP toxicity in LLC tumor-bearing mice. These findings suggested that JC-001 is a potential clinical application that 
may avoid the side effect with high dose CDDP in CDDP-based chemotherapeutic regimens.

\section{Additional files}

Additional file 1: JC-001 reduced the tumor mass in BALB/C nude immunodeficient mice inoculated with LLC1 subcutaneously. The average tumor weight in the $3 \mathrm{X} \mathrm{JC-001-treated} \mathrm{group}(n=8)$ was significantly reduced to $39 \%$ of that in the control group $(n=8) .{ }^{* *} p<0.01$ compared with the control group. (DOCX 67kb)

Additional file 2: Relative cytokine levels in the tumor microenvironment (A) and serum (B) in the JC-001-treated group compared with the control group. LLC1 tumor-bearing mice were treated with 3X JC-001 in $\mathrm{H} 2 \mathrm{O}$ for 23 days, and cytokine levels in tumor tissues were analyzed by RayBio ${ }^{\oplus}$ mouse cytokine antibody array 3.1. (DOCX $260 \mathrm{~kb})$

\section{Abbreviations \\ ADCA: Adenocarcinoma; ALT: Alanine aminotransferase; AST: Aspartate transaminase; CDDP: cis-diamminedichloroplatinum (II); CX3CL1: Fractalkine; CXCL5: C-X-C motif chemokine 5; FasL: Fas ligand; H\&E: Hematoxylin and eosin; IFN-y: Interferon gamma; IHC: Immunohistochemistry; IL: Interleukin; MHC-I: Class I major histocompatibility complex; MTT: 3-(4,5-dimethylthiazol- 2-yl)-2,5-diphenyltetrazolium bromide; NK: Natural killer; NSCLC: Non-small- cell lung cancer; SCCA: Squamous cell carcinoma; SCF: Stem cell factor; SCLC: Small-cell lung cancer; Th1: T helper type I; Th17: T helper type 17; TNF-a: Tumor necrosis factor alpha}

\section{Acknowledgments}

We are very grateful to a professor Chung-Hung Tsai for histological analysis.

\section{Funding}

This study was supported by Grant 99-CSMU36 from Chung Shan Medical University, Taiwan

\section{Availability of data and materials}

All data of this study is included in this article.

\section{Authors' contributions}

MHC, MSJ and JHT performed the laboratory work, conducted the statistical analysis and were major contributors to the writing of the manuscript. MSJ provided academic recommendations regarding immunology. FJL and JTC provided advice regarding research questions and technological support. All authors read and approved the final manuscript.

\section{Competing interests}

The authors declare that they have no competing interests.

\section{Consent for publication}

Not applicable.

\section{Ethics approval and consent to participate}

All the experimental procedures were approved by the Institutional Animal Care and Use Committee of Chung Shan Medical University.

\section{Publisher's Note}

Springer Nature remains neutral with regard to jurisdictional claims in published maps and institutional affiliations.

\section{Author details}

'Institute of Medicine, Chung Shan Medical University, No. 110, Sec. 1 , Jianguo N. Rd, Taichung City 40201, Taiwan. ${ }^{2}$ Institute of Biochemistry, Microbiology and Immunology, Chung Shan Medical University, No.110, Sec.1, Jianguo N. Rd, Taichung City 40201, Taiwan. ${ }^{3}$ Immunology Research Center, Chung Shan Medical University, No. 110, Sec. 1, Jianguo N. Rd, Taichung City 40201, Taiwan. ${ }^{4}$ Division of Allergy, Immunology and Rheumatology, Department of Internal Medicine, Chung Shan Medical University Hospital, 110, Sec.1, Jianguo N. Rd, Taichung City 40201, Taiwan.
${ }^{5}$ Department of Chest Medicine Chung Shan Medical University Hospital, No. 110, Sec. 1, Jianguo N. Rd, Taichung City 40201, Taiwan. 'Department of Medical Research, Chung Shan Medical University Hospital, No. 110, Sec. 1, Jianguo N. Rd, Taichung City 40201, Taiwan.

Received: 14 January 2017 Accepted: 5 April 2017

Published online: 11 April 2017

\section{References}

1. Siegel R, Ma J, Zou Z, Jemal A. Cancer statistics, 2014. CA Cancer J Clin. 2014;64:9-29.

2. Travis WD, Brambilla $E$, Noguchi $M$, Nicholson AG, Geisinger $K$, Yatabe Y, Powell CA, Beer D, Riely G, Garg K, Austin JH, Rusch WW, Hirsch FR, Jett J, Yang PC, Gould M, American Thoracic Society. International Association for the Study of Lung Cancer/American Thoracic Society/ European Respiratory Society: international multidisciplinary classification of lung adenocarcinoma: executive summary. Proc Am Thorac Soc. 2011:8:381-5.

3. Sun S, Schiller JH, Gazdar AF. Lung cancer in never smokers-a different disease. Nat Rev Cancer. 2007:7:778-90.

4. Houghton AM. Mechanistic links between COPD and lung cancer. Nat Rev Cancer. 2013:13:233-45.

5. Spira A, Beane J, Shah V, Liu G, Schembri F, Yang X, Palma J, Brody JS. Effects of cigarette smoke on the human airway epithelial cell transcriptome. Proc Natl Acad Sci U S A. 2004;101:10143-8.

6. Mao L, Lee JS, Kurie JM, Fan YH, Lippman SM, Lee JJ, Ro JY, Broxson A, Yu R, Morice RC, Kemp BL, Khuri FR, Walsh GL, Hittelman WN, Hong WK. Clonal genetic alterations in the lungs of current and former smokers. J Natl Cancer Inst. 1997;89:857-62

7. Lebwohl D, Canetta R. Clinical development of platinum complexes in cancer therapy: an historical perspective and an update. Eur J Cancer. 1998; 34:1522-34

8. Chirino Yl, Pedraza-Chaverri J. Role of oxidative and nitrosative stress in cisplatin-induced nephrotoxicity. Exp Toxicol Pathol. 2009;61:223-42.

9. Lorusso D, Petrelli F, Coinu A, Raspagliesi F, Barni S. A systematic review comparing cisplatin and carboplatin plus paclitaxel-based chemotherapy for recurrent or metastatic cervical cancer. Gynecol Oncol. 2014;133:117-23.

10. Hagihara A, Ikeda M, Ueno H, Morizane C, Kondo S, Nakachi K, Mitsunaga S, Shimizu S, Kojima Y, Suzuki E, Katayama K, Imanaka K, Tamai C, Inaba Y, Sato Y, Kato M, Okusaka T. Phase I study of combination chemotherapy using sorafenib and transcatheter arterial infusion with cisplatin for advanced hepatocellular carcinoma. Cancer Sci. 2014;105:354-8.

11. Herbst RS, Arquette M, Shin DM, Dicke K, Vokes EE, Azarnia N, Hong WK, Kies MS Phase II multicenter study of the epidermal growth factor receptor antibody cetuximab and cisplatin for recurrent and refractory squamous cell carcinoma of the head and neck. J Clin Oncol. 2005:23:5578-87.

12. Chuang M-H, Chang JT, Hsu L-J, Jan M-S, Lu F-J. Antitumor activity of the Chinese medicine JC-001 is mediated by immunomodulation in a murine model of hepatocellular carcinoma. Integr Cancer Ther. 2016. doi:10.1177/ 1534735416664173

13. Hyakudomi M, Matsubara T, Hyakudomi R, Yamamoto T, Kinugasa S, Yamanoi A, Maruyama R, Tanaka T. Increased expression of fractalkine is correlated with a better prognosis and an increased number of both CD8+ T cells and natural killer cells in gastric adenocarcinoma. Ann Surg Oncol. 2008:15:1775-82.

14. Park MH, Lee JS, Yoon JH. High expression of CX3CL1 by tumor cells correlates with a good prognosis and increased tumor-infiltrating CD8+ T cells, natural killer cells, and dendritic cells in breast carcinoma. J Surg Oncol. 2012;106:386-92.

15. Rengarajan J, Szabo SJ, Glimcher LH. Transcriptional regulation of Th1/Th2 polarization. Immunol Today. 2000;21:479-83.

16. Rossi D, Zlotnik A. The biology of chemokines and their receptors. Ann Rev Immunol. 2000;18:217-42.

17. Sallusto F, Lanzavecchia A, Mackay CR. Chemokines and chemokine receptors in T-cell priming and Th1/Th2-mediated responses. Immunol Today. 1998;19:568-74.

18. Yoneda O, Imai T, Nishimura M, Miyaji M, Mimori T, Okazaki T, Domae N, Fujimoto $\mathrm{H}$, Minami $Y$, Kono T, Bloom ET, Umehara $\mathrm{H}$. Membrane-bound form of fractalkine induces IFN-gamma production by NK cells. Eur J Immunol. 2003:33:53-8.

19. Chin YE, Kitagawa M, Su WC, You ZH, Iwamoto Y, Fu XY. Cell growth arrest and induction of cyclin-dependent kinase inhibitor p21 WAF1/CIP1 mediated by STAT1. Science. 1996;272:719-22. 
20. Dimco G, Knight RA, Latchman DS, Stephanou A. STAT1 interacts directly with cyclin D1/Cdk4 and mediates cell cycle arrest. Cell Cycle. 2010;9:4638-49.

21. O'Connell J, Bennett MW, Nally K, O'Sullivan GC, Collins JK, Shanahan F. Interferon-gamma sensitizes colonic epithelial cell lines to physiological and therapeutic inducers of colonocyte apoptosis. J Cell Physiol. 2000;185:331-8.

22. Tura BJ, Bunyan KE, Harrison DJ. The effect of IFNgamma on the hepatocyte: cell cycle and apoptosis. Int J Exp Pathol. 2001;82:317-26.

23. Martini M, Testi MG, Pasetto M, Picchio MC, Innamorati G, Mazzocco M, Ugel S, Cingarlini S, Bronte V, Zanovello P, Krampera M, Mosna F, Cestari T, Riviera AP, Brutti N, Barbieri O, Matera L, Tridente G, Colombatti M, Sartoris S. IFN-gammamediated upmodulation of MHC class I expression activates tumor-specific immune response in a mouse model of prostate cancer. Vaccine. 2010;28:3548-57.

24. Ando S, Catalano S. The multifactorial role of leptin in driving the breast cancer microenvironment. Nat Rev Endocrinol. 2012;8:263-75.

25. Fernandez-Riejos P, Najib S, Santos-Alvarez J, Martin-Romero C, Perez-Perez A, Gonzalez-Yanes C, Sanchez-Margalet V. Role of leptin in the activation of immune cells. Mediat Inflamm. 2010;2010:568343.

26. Lang K, Ratke J. Leptin and Adiponectin: new players in the field of tumor cell and leukocyte migration. Cell Commun Signal. 2009;7:27.

27. Maya-Monteiro CM, Bozza PT. Leptin and mTOR: partners in metabolism and inflammation. Cell Cycle. 2008;7:1713-7.

28. Zheng Q, Dunlap SM, Zhu J, Downs-Kelly E, Rich J, Hursting SD, Berger NA, Reizes $\mathrm{O}$. Leptin deficiency suppresses MMTV-Wnt-1 mammary tumor growth in obese mice and abrogates tumor initiating cell survival. Endocr Relat Cancer. 2011:18:491-503.

29. Kuo PL, Chen YH, Chen TC, Shen KH, Hsu YL. CXCL5/ENA78 increased cell migration and epithelial-to-mesenchymal transition of hormoneindependent prostate cancer by early growth response-1/snail signaling pathway. J Cell Physiol. 2011;226:1224-31.

30. Miyazaki H, Patel V, Wang H, Edmunds RK, Gutkind JS, Yeudall WA. Downregulation of CXCL5 inhibits squamous carcinogenesis. Cancer Res. 2006;66: 4279-84.

31. Kawamura M, Toiyama Y, Tanaka K, Saigusa S, Okugawa Y, Hiro J, Uchida K, Mohri Y, Inoue Y, Kusunoki M. CXCL5, a promoter of cell proliferation, migration and invasion, is a novel serum prognostic marker in patients with colorectal cancer. Eur J Cancer. 2012;48:2244-51.

32. Li A, King J, Moro A, Sugi MD, Dawson DW, Kaplan J, Li G, Lu X, Strieter RM, Burdick M, Go VL, Reber HA, Eibl G, Hines OJ. Overexpression of CXCL5 is associated with poor survival in patients with pancreatic cancer. Am J Pathol. 2011:178:1340-9.

33. Zhou SL, Dai Z, Zhou ZJ, Wang XY, Yang GH, Wang Z, Huang XW, Fan J, Zhou J. Overexpression of CXCL5 mediates neutrophil infiltration and indicates poor prognosis for hepatocellular carcinoma. Hepatology. 2012;56:2242-54.

34. Gorelik E, Lokshin A, Levina V. Lung cancer stem cells as a target for therapy. Anti Cancer Agents Med Chem. 2010;10:164-71.

35. Bellone G, Carbone A, Sibona N, Bosco O, Tibaudi D, Smirne C, Martone T, Gramigni C, Camandona M, Emanuelli G, Rodeck U. Aberrant activation of c-kit protects colon carcinoma cells against apoptosis and enhances their invasive potential. Cancer Res. 2001;61:2200-6.

36. Yasuda A, Sawai H, Takahashi H, Ochi N, Matsuo Y, Funahashi H, Sato M, Okada Y, Takeyama H, Manabe T. The stem cell factor/c-kit receptor pathway enhances proliferation and invasion of pancreatic cancer cells. Mol Cancer. 2006:5:46

37. Zhang M, Ma Q, Hu H, Zhang D, Li J, Ma G, Bhat K, Wu E. Stem cell factor/ c-kit signaling enhances invasion of pancreatic cancer cells via HIF-1alpha under normoxic condition. Cancer Lett. 2011;303:108-17.

38. Huang B, Lei Z, Zhang GM, Li D, Song C, Li B, Liu Y, Yuan Y, Unkeless J, Xiong $\mathrm{H}$, Feng ZH. SCF-mediated mast cell infiltration and activation exacerbate the inflammation and immunosuppression in tumor microenvironment. Blood. 2008;112:1269-79.

39. Wei JJ, Song CW, Sun LC, Yuan Y, Li D, Yan B, Liao SJ, Zhu JH, Wang Q, Zhang GM, Feng ZH. SCF and TLR4 ligand cooperate to augment the tumor-promoting potential of mast cells. Cancer Immunol Immunother. 2012;61:303-12.

40. Jelly ND, Hussain II EJ, Eremin O, El-Sheemy M. The stem cell factor antibody enhances the chemotherapeutic effect of adriamycin on chemoresistant breast cancer cells. Cancer Cell Int. 2012;12:21.

41. Lu C, Hassan HT. Human stem cell factor-antibody [anti-SCF] enhances chemotherapy cytotoxicity in human CD34+ resistant myeloid leukaemia cells. Leuk Res. 2006;30:296-302.
42. Middleton GW, Annels NE, Pandha HS. Are we ready to start studies of Th17 cell manipulation as a therapy for cancer? Cancer Immunol Immunother. 2012;61:1-7.

43. Martin F, Apetoh L, Ghiringhelli F. Controversies on the role of Th17 in cancer: a TGF-beta-dependent immunosuppressive activity? Trends Mol Med. 2012;18:742-9.

44. Ouyang W, Kolls JK, Zheng Y. The biological functions of Thelper 17 cell effector cytokines in inflammation. Immunity. 2008;28:454-67.

45. Gregory AD, Houghton AM. Tumor-associated neutrophils: new targets for cancer therapy. Cancer Res. 2011;71:2411-6.

46. Ramakrishnan R, Assudani D, Nagaraj S, Hunter T, Cho HI, Antonia S, Altiok S, Celis E, Gabrilovich DI. Chemotherapy enhances tumor cell susceptibility to CTL-mediated killing during cancer immunotherapy in mice. J Clin Invest. 2010;120:1111-24.

47. Antonia SJ, Mirza N, Fricke I, Chiappori A, Thompson P, Williams N, Bepler G, Simon G, Janssen W, Lee JH, Menander K, Chada S, Gabrilovich DI. Combination of p53 cancer vaccine with chemotherapy in patients with extensive stage small cell lung cancer. Clin Cancer Res. 2006;12:878-87.

48. Arlen PM, Gulley JL, Parker C, Skarupa L, Pazdur M, Panicali D, Beetham P, Tsang KY, Grosenbach DW, Feldman J, Steinberg SM, Jones E, Chen C, Marte J, Schlom J, Dahut W. A randomized phase II study of concurrent docetaxel plus vaccine versus vaccine alone in metastatic androgen-independent prostate cancer. Clin Cancer Res. 2006;12:1260-9.

\section{Submit your next manuscript to BioMed Central and we will help you at every step:}

- We accept pre-submission inquiries

- Our selector tool helps you to find the most relevant journal

- We provide round the clock customer support

- Convenient online submission

- Thorough peer review

- Inclusion in PubMed and all major indexing services

- Maximum visibility for your research

Submit your manuscript at www.biomedcentral.com/submit
) Biomed Central 